\title{
Research and Development of Competitive Wushu Competition Management platform based on Internet of things Technology
}

\author{
Guofang Kuang ${ }^{1, a^{*}}$ and Chunlin Kuang ${ }^{1}$ \\ ${ }^{1}$ Luoyang Normal University, Luoyang, 471934, China \\ akuangguofang2012@163.com \\ * The corresponding author
}

Keywords: Internet of things; RFID; Face detection; Competition management; Wushu judgment

\begin{abstract}
The paper introduces the structure, working principle and function of each part of the system, and discusses in detail the realization of the software part of the system. Compared with other systems, the system has the advantages of lower cost, more convenient, stronger ability of data analysis, and can be used in all levels of competitive martial arts competitions. The system will make the scoring of martial arts competitions more transparent and fair. It can greatly improve the enthusiasm of the people to participate in the competition. The paper presents research and development of competitive Wushu competition management platform based on Internet of things technology.
\end{abstract}

\section{Introduction}

The judges give code or score synchronously by using a grader when athlete is playing . For example, an athlete's heel is off the ground when he sweeps his legs in option long fist. At least 2 of the three judges enter the code of 21 on the grader within 3 seconds before a deduction be made. This method of scoring synchronously with the performance of athletes has not only changed the previous judges' note-taking scoring operation, shortened the scoring time, reduced some tedious manual operation methods and forms, but also improved the working efficiency [1]. At the same time, it avoids the phenomenon of turning over the wrong score and reversing the score, and greatly reduces the possibility of the judge's bad subjective intention to control the score result, and increases the transparency of the judgment.

This is useful for construction of judge team. The electronic scoring system puts forward higher requirements for the criterion of judges' scoring technique, and it is beneficial to the promotes the development of martial arts movement towards the direction of standardization.

The traditional method of constructing competitive martial arts scoring system is to use computers or self-made scoring devices. These things are connected by wire. This System is not only complicated to arrange on the spot, but also prone to produce various problems that lead to the interruption of the competition. With the development of technology about internet of things, hand-hold mobile terminal is widely used in people's production and life.

In this paper, the Wushu management platform is studied by combining theory with practice, computer software simulation and practical system. Firstly, this paper studies the existing rules of Wushu judgment, then synthetically analyzes the advantages and disadvantages of the existing management system, studies the feasibility of the new management system, and then carries on the experiment and simulation to the key technology of the management system, and designs the overall structure of the system. The system database is designed, detailed design is carried out, and an extensible and reliable management platform is constructed. The reliability of the system is preliminarily verified by computer simulation and comparison, and tested by real environment.

\section{Key Technology Research}

The parity check is simple and widely used. The longitudinal redundancy check algorithm is mainly used to quickly check small data blocks. Although the cyclic redundancy check method can not correct the error, it can identify the transmission error with high reliability. In addition, the channel coding of data 
can improve the ability of anti-interference, error-detection and error-correcting in the process of data transmission. In many cases, there are many tags which wait to be identified in the scope of the reader. One of the advantages of RFID system is that the reader can identify multiple tags in a very short time [2]. The communication from reader to tag is similar to the broadcasting of radio. Multiple tags receive data streams sent by the same reader at the same time. In order to prevent multiple tag data from interfering with each other in the receiver of reader and writer, anti-collision method must be adopted to solve the problem.

The RFID system as shown in figure 1 generally consists of three basic parts: tag, reader and central information system. The tag and the reader transmit data in a non-contact manner, and the database is wired to the reader. The tag is made up of a coupled antenna and a chip. Each tag has a unique electronic product code (EPC) and is attached to the object or object being identified. The reader is connected to an external antenna by a device that reads or erases label information. The central information system (or database for short) includes middle-ware, information processing system, database and so on, which is used to process the information read from tag by the reader. These functions include the implementation of information encryption, security authentication is shown in Fig. 1.

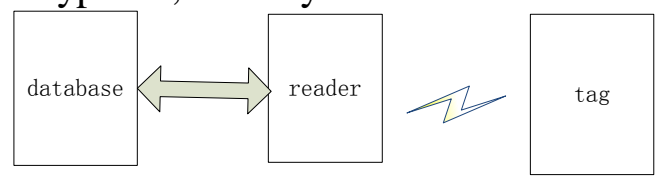

Figure 1. Finite RFID system

The frequency usually used by the reader when RF signals is send called the operating frequency of the RFID system, which can be divided into three ranges: low frequency $(30 \sim 300 \mathrm{kHz})$, high frequency $(3 \sim 30 \mathrm{MHz})$ and $\mathrm{UHF}(300 \mathrm{MHz} \sim 3 \mathrm{GHz})$. Low frequency and intermediate frequency are mainly suitable for short distance and low cost applications [3]. The high frequency system is suitable for situations where the recognition distance is long, the rate of reading and writing data is high. The common working frequencies are low frequency $125 \mathrm{kHz}$, high frequency $13.56 \mathrm{MHz}$ and ultra high frequency $915 \mathrm{MHz}$, etc. Normally, the higher the frequency, the faster the speed of reading and writing is produced, the more efficient the data is transmitted.

In addition, in these two systems, the energy transmission from reader to transponder is continuous and independent of the direction of data transmission. The data transfer from reader to tag may cause the failure of recognizing the tag properly or to judging one label as another.In RFID system, the anti-interference methods can be used as follows: data check and channel coding. Through the data integrity check method, the system can check out the data that has been interfered, and the most commonly used method is parity check, longitudinal redundancy check and cyclic redundancy check, etc. For parity check,in order to ensure that the transmitter and receiver use the same method, parity check or parity check must be decided before data transmission.

Face detection is very important in the process of processing, and it has a very important influence on the subsequent feature extraction and matching. At present, face detection algorithms are constantly updated and upgraded, and important progress has been made in the research of face detection and recognition with different skin color and different pose.

In fact, face matching is to classify and recognize the extracted face features. The features of the stored face images are used for classification and training. The face images which are not stored need to be matched by the similarity calculation through this classifier.

\section{Wushu Management System based on Internet of Things Meeting the Requirements of Competition}

The system adopts WLAN networking technology based on Wi-Fi and wireless technology to construct network, which saves the link of match field wiring and is very convenient to use. The existing referee platform is wired; now need to set a large number of network lines, time-consuming and laborious. 
However, using WLAN technology increases the complexity of system development and requires multiple technologies to maintain reliable data communication.

From the view of the whole process of face recognition, every module has direct or indirect influence on face recognition. The algorithm of each module needs to be considered synthetically. The feature extraction algorithm has an important effect on the correct face matching. Different types of feature selection will also be affected by the image clarity and noise [4]. The goal of this paper is to complete a face recognition authentication system, which needs to consider the actual effects, data processing conditions, environment and other practical factors, considering comprehensively, using the individual specificity of LBP features for face recognition. Considering the individual specificity of LBP feature, face recognition is carried out.

The software module of the system consists of 9 parts. The software structure is shown in Fig. 2.

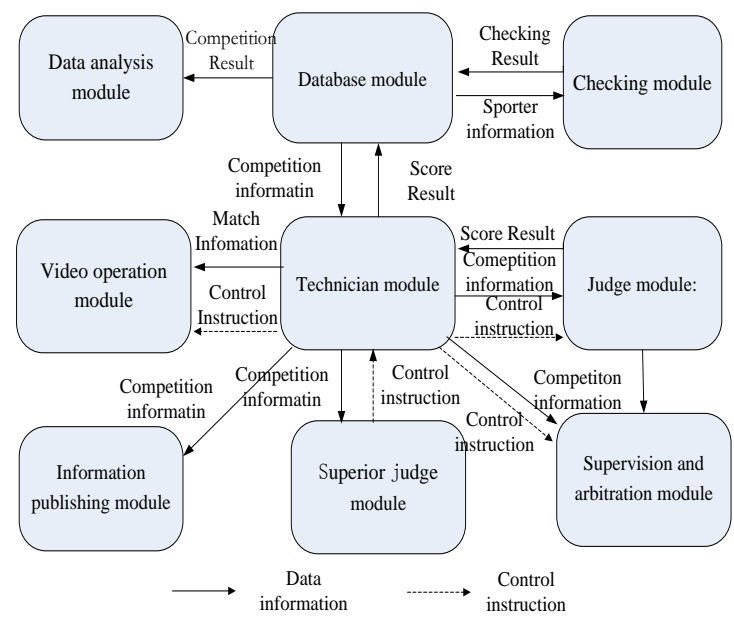

Figure 2. Finite Software structure diagram

Database module: the database module runs in the data center. Competition information, such as the standard of marks, the action specification error code and its values of group A, the practice level arrangement error code and its values of group B, the $\mathrm{C}$ group difficulty level code and its values of group $\mathrm{C}$, calculation rules and the score of athlete are stored .

Technician module: the module runs on the technician equipment, operated by the professional technicians under the command of the judge. After the program run, the item information of the current athlete is read from the database [5]. Before the start of the match, it is sent to the judge module, superior judge module and the video module, etc. When the game starts and ends, the technician module sends the start and end instructions to them. When the judge thinks that the result can be effectively released, the technology sends the information release instruction to the information publishing module, as is shown by equation (1).

$$
\mathrm{s}^{2}=\frac{1}{\mathrm{n}(\mathrm{m}-1)} \sum_{\mathrm{i}=1}^{\mathrm{m}} \sum_{\mathrm{j}=1}^{\mathrm{n}}\left(\mathrm{x}_{\mathrm{ij}}-\mathrm{t}_{\mathrm{j}}\right)^{2}
$$

Judge module: this module runs on the judge terminal equipment. Convenient and easy using is the main problem to consider. This module receives the match information, the instructions for the start and end of the match from the technician, and sends score from judge to the technician module.

Supervision and arbitration module: this module runs on the supervision and arbitration equipment, receives the athlete information sent by the technician and the real-time marking information from the judge transmitted by the technician. The supervisor and arbitration can monitor the competition through this module, and can quickly find the problems in the course of the competition.. 
RFID is a widely used data input device in the Internet of things system. It can be used to realize fast non-contact identification [6]. The domestic ID card is a typical RFID whose frequency is $13.56 \mathrm{MHz}$ and 14443B protocol is adopted. In the registration stage of the competition, information collection can be realized only by using identity in a light sweep, or easily through the mobile phone APP.

By using the Internet of things technology, the video information of the competition, the announcement of the competition, the result of the athletes and so on are fused. The information can be obtained through the APP of computer or mobile phone.

\section{Research and Development of Competitive Wushu Competition Management Platform based on Internet of things Technology}

On the basis of studying the relationship between Wushu technology and Wushu, fair and just electronic scoring mechanism and reliable real time communication and trusted data model under WiFi environment, the sports management system is constructed by using Android C\#VS2010 and other software development technologies [7]. Complete Wushu competition management system based on Internet of things technology is realized from athlete registration, spot scoring to final result, ranking announcement. Provide a full range of information technology support for a competition.

Information publishing module: after receiving the athlete information sent by the technician module, the module displays the current athlete information on the large screen. When the final score and the score of group A, group B or group $\mathrm{C}$ are received will be displayed. The module can also publish the results to the staff or athlete through web query.

Superior judge module: this module runs in the superior judge equipment, receives the athlete information sent by the technician in real time and the real-time marking information of the judge transmitted by the technician equipment. Through this module, the superior judge can supervise the judgment of the judge and modify the score of a certain part directly within the limits of the competition rules.

Video operation module: this module runs on the video computer, records the video according to the athlete information sent by the technician module, and stores the result of the judge's scoring about the athlete. These stored scoring results can be helpful for future video review and video learning.

Data analysis module: the data analysis module mainly analyzes the conformance degree between the routine movement and the standard movement, and provides the reference for the later training.

Checking module: this module reads the identity card information of the participants through RFID technology, at the same time, obtains the head images of the participants through the camera, and realizes the automatic checking through face recognition technology. The whole analysis of action specification error: the category of action specification error in group A is summarized, and the distribution of action specification error in this competition is obtained, as shown in Fig. 3.

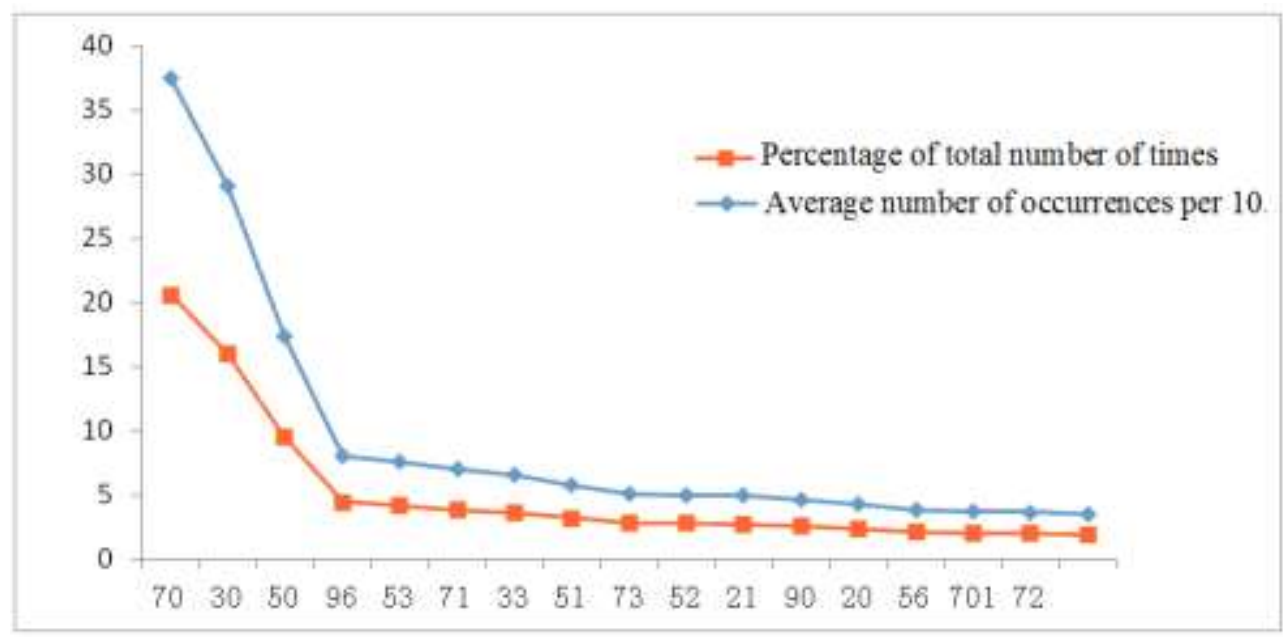

Figure 3. Finite Action specification error analysis diagram 
By observing the deviation analysis chart, we can clearly find that the referee group that judges the female Cangchang fist is the one with the least fluctuation in the score of the four referee groups, that is, the stability and consistency of the score are relatively high, and the scoring level is relatively good. The judges who judged the quality of male armour were obviously the worst of the four groups.

The consistency of the result of judging the action quality is to investigate whether the judges of the whole group are consistent with the overall score of the participating athletes. The standard deviation analysis can be used to evaluate the level (consistency of scoring results) of different referees in a match.

\section{Summary}

For athletes, the traditional evaluation methods rely on the expert will and weight system too much, so the subjective color is strong, and the evaluation result is unfair. In the design of the system, the athlete selection evaluation model based on the sudden change series is optimized. Based on the catastrophe theory, the mutation type attributes of the index system are deeply excavated, and the comprehensive evaluation is carried out by using the original measurement data and the normalized formula. In order to reduce the subjectivity of evaluation, it is more simple and easy to carry out.

\section{Acknowledgements}

This paper is supported by Science and Technology Key Project of Henan Province (162102310478), and by School Applied Science and Technology Research Foundation 2017 "Research on the Application of Wushu (routine) Intelligent Competition arrangement system based on SVM-KNN".

\section{References}

[1] Seung-Jung Shin, Jung-Hwa Kang, and Kae-Dal Kwack. A Study of a Protocol Model for Information Security of the RFID Base Individual Identification Device, RNIS, Volume 7, pp. 10 12, 2011.

[2] Yan Bo, Development of traceability platform for aquatic product supply chain based on RFID and EPC, Journal of Agricultural Engineering. 2013,29(5).

[3] Joe Kman, Wang Guiran, Sun Wei, et al, Perceived Sports: the Application Architecture of Internet of things Technology in Sports, Journal of Wuhan Institute of physical Education. 2012, 46(1).

[4] Zhang Hailiang, The birth of competitive Wushu, Shanghai: Shanghai Institute of physical Education.2010.

[5] Kuang Guofang, The Application of Management Information System in Internet of Things based on RFID Technology,Sensors\&Transducers. 2013.7.

[6] Joseph K. Siror, Liang Guanqun, Kaifang Pang, Sheng Huanye, Wang Dong. Use of RFID for Intelligent Pre-shipment Inspection, JDCTA, Vol. 4, No. 8, pp. $242 \sim 251,2010$.

[7] Du Jin. Application of "Internet of Things" in Electronic Commerce, JDCTA, Vol. 6, No. 8, pp. 222 $\sim 230,2012$. 